Eastern Illinois University

The Keep

Faculty Research and Creative Activity

Kinesiology \& Sports Studies

November 2014

\title{
Identification of high responders for interleukin-6 and creatine kinase following acute eccentric resistance exercise in elderly obese women
}

Vitor Tajra

Catholic University of Brasilia

Ramires Alsamir Tibana

Catholic University of Brasilia

Denis Cesar Leite Vieira

Catholic University of Brasilia

Darlan Lopes de Farias

Catholic University of Brasilia

Tatiane Gomes Teixeira

Catholic University of Brasilia

See next page for additional authors

Follow this and additional works at: http://thekeep.eiu.edu/kss_fac

Part of the Kinesiology Commons

\section{Recommended Citation}

Tajra, Vitor; Tibana, Ramires Alsamir; Leite Vieira, Denis Cesar; de Farias, Darlan Lopes; Teixeira, Tatiane Gomes; Funghetto, Silvana Schwerz; Silva, Alessandro Oliviera; de Sousa, Nuno Manuel Frade; Willardson, Jeffrey; Oliviera Karnikowski, Margo Gomes; and Prestes, Jonato, "Identification of high responders for interleukin- 6 and creatine kinase following acute eccentric resistance exercise in elderly obese women" (2014). Faculty Research and Creative Activity. 35.

http://thekeep.eiu.edu/kss_fac/35

This Article is brought to you for free and open access by the Kinesiology \& Sports Studies at The Keep. It has been accepted for inclusion in Faculty Research and Creative Activity by an authorized administrator of The Keep. For more information, please contact tabruns@eiu.edu. 


\section{Authors}

Vitor Tajra, Ramires Alsamir Tibana, Denis Cesar Leite Vieira, Darlan Lopes de Farias, Tatiane Gomes Teixeira, Silvana Schwerz Funghetto, Alessandro Oliviera Silva, Nuno Manuel Frade de Sousa, Jeffrey Willardson, Margo Gomes Oliviera Karnikowski, and Jonato Prestes 


\title{
Identification of high responders for interleukin- 6 and creatine kinase following acute eccentric resistance exercise in elderly obese women
}

Vitor Tajra, Ramires Alsamir Tibana, Denis Cesar Leite Vieira, Darlan Lopes de Farias, Tatiane Gomes Teixeira, Silvana Schwerz Funghetto, Alessandro Oliveira Silva, Nuno Manuel Frade de Sousa, Jeffrey Willardson, Margô Gomes Oliveira Karnikowski, Jonato Prestes

\begin{abstract}
A b stract
Objectives: Resistance exercise is used as a non-pharmacological tool to elicit both gains in and maintenance of physical function in the elderly. Thus, the present study examined the acute response of creatine kinase and interleukin- 6 following an eccentric resistance exercise session in elderly obese women classified as high responders or normal responders.
\end{abstract}

Design: Cross-sectional field study.

Methods: Ninety elderly obese women $(69.4 \pm 6.01$ years) were tested for a 10 repetition maximum on the leg extension exercise and then completed an acute eccentric resistance exercise session consisting of seven sets of 10 repetitions at $110 \%$ of 10 repetition maximum with a rest of 3 min between sets. Subjects were divided into normal response or high response on the basis of the peak serum interleukin-6 ( $\mathrm{NR}=59$ and $\mathrm{HR}=7$ ) and creatine kinase ( $\mathrm{NR}=81$ and $\mathrm{HR}=9)$ concentration being greater than (HR) or less than (NR) the 90th percentile.

Results: Creatine kinase was higher at $0 \mathrm{~h}, 3 \mathrm{~h}, 24 \mathrm{~h}$ and $48 \mathrm{~h}$ following the ERE for the HR group. The peak creatine kinase was significantly higher in HR group versus the normal response group. The average increase in the serum interleukin- $6 \Delta$ for the HR group $(\sim 850 \%)$ was significantly higher versus the normal response group $(\sim 55 \%)$. Serum interleukin-6 was significantly higher at $0 \mathrm{~h}$ and $24 \mathrm{~h}$ following eccentric resistance exercise only for the high response group, while peak levels were significantly higher in high response group versus the normal response group ( $p \leq 0.005$ ). Only one subject met the criteria to be classified as high response for both creatine kinase and interleukin- 6 responsiveness.

Conclusions: Elderly individuals classified as high response experienced greater creatine kinase and interleukin- 6 responses to ERE. Thus, a prudent approach for eccentric resistance exercise prescription might be programming additional recovery days and/or lower intensity training, especially in the beginning stages of a program.

\section{Introduction}


Resistance exercise (RE) is used as a non-pharmacological tool to elicit both gains in and maintenance of physical function in the elderly; specific RE training methods need further exploration todetermine the best application and the risks for this population.1It is well known that RE can lead to muscle microtrauma, which is an important stimulus for muscular growth.2,3 Creatine kinase (CK) concentration has been measured to assess the extent of muscle microtrauma following RE.4,5 It was noted in previous research that some individuals expressed disproportionately greater $\mathrm{CK}$ con-centration following the same volume of $\mathrm{RE}$ and were categorized as high responders (HR).4,5

It has been shown that eccentric exercise can induce greater muscle damage as indicated by greater CK concentration.6 More-over, there is an association between the acute inflammatory response and the muscle damage induced by eccentric exercise.7 It is known that myofibers express interleukin-6 (IL-6), a key cytokine expressed in the inflammatory response, which is involved with satellite cell proliferation, differentiation and muscle repair.8,9 Considering this, it would be of great interest to investigate whether individuals may express different IL-6 responsiveness following a given RE stimulus, as was previously demonstrated for CKconcentration. 4,5

Interestingly, elderly individuals preserve eccentric strength more readily than concentric strength.10 Evidence suggests that eccentric resistance exercise (ERE) can be an excellent method to utilize during the initial stages of a resistance training program in the elderly10,11; due to the lower perceived exertion versus conventional resistance exercise.12 The higher efficiency of eccentric resistance exercise (i.e. intense muscle work is achieved at a lower metabolic expense), 13 renders it as a powerful tool for restoring muscle strength in people with a limited capacity to train at high intensities such as older adults.14 Thus, investigating the acuteCK and IL- 6 responses to eccentric resistance exercise in older adults, and the potential for individuals to express different levels of responsiveness for these markers, could provide valuable information for the prescription of RE training for this population.

To the best of our knowledge, no study has investigated CK concentration and IL-6 responses following eccentric resistance exercise in older obese individuals, and further assessed the possible existence of differing responsiveness in the IL-6 marker. Thus, the purpose of the present study was to examine CK and IL6 concentration responses following eccentric resistance exercise in elderly obese women, and to assess the existence of high responders and normal responders (NR) for these markers. Our hypothesis was that serum CK and IL- 6 would be higher following eccentric resistance exercise for those individuals classified as high responders, and there would be no significant increase in CK and IL-6 in elderly individuals classified as normal responders.

\section{Methods}


Ninety elderly women from a local community (69.4 \pm 6.01 years of age, $152.6 \pm 6.2$ $\mathrm{cm}$ in height, body mass of $64.6 \pm 12.1 \mathrm{~kg}$,lean mass of $58.8 \pm 5.5 \%$, and body fat of $41.2 \pm 5.5 \%$ ) were recruited to participate in the present study on a voluntary basis. Individuals were included according to the following criteria: age $\geq 60$ years, sedentary elderly females, body fat percentage $>32 \%$ and completion of all testing procedures. The list of medications used by the individuals included omeprazole (gastrointestinal tract/metabolism), losartan (cardiovascular system), levotiroxin, furosemide (diuretic) and simvastatin (cholesterol control). Obesity was determined as recommended by the National Instituteof Diabetes and Digestive and Kidney Diseases 15, assuming a cutoff point of 32\% for women. Sedentary state was evaluated by the International Physical Activity Questionnaire. Women with inflammatory, rheumatic, or autoimmune conditions or use of medications (i.e., beta blockers, hormone replacement therapy, selective estrogen receptor modulators, anti-inflammatory, insulin, fish oil and multivitamin supplements) that could modulate the biochemical response to RE were excluded. The study was approved by the Institutional Research Ethics Committee (protocol\#035/2011), and all subjects gave written, informed consent.

High responders for $\mathrm{CK}$ concentration were defined as a $\Delta \mathrm{CK} \geq 90$ th percentile or $96.3 \mathrm{U} / \mathrm{l}$, according to the definition of a "rare event" as compared with normal responders.16,17 Accordingto Toft et al., 18plasma IL-6 concentrations increased approximately four-fold immediately following eccentric muscle actions in young subjects (20-27 years); whereas, the increase was much smaller in elderly subjects (67-75 years) (approximately 90\% increase following eccentric exercise consisting of $60 \mathrm{~min}$ of opposing the rotation of cycle ergometer pedals down to $60 \mathrm{rpm}$ ). In this way, we assumed that the $\Delta \mathrm{IL}-6 \geq 90$ th percentile or $7.5 \mathrm{pg} / \mathrm{ml}$ was a valid criterion to define HR for this marker.

A ten repetition-maximum (10 RM) test was performed accord-ing to the recommendations of Tibana et al.19. On the first visit, subjects completed a medical form and physical questionnaire, anthropometric measures, dual-energy X-ray absorptiometry(DXA, General Electric-GE model 8548 BX1L, 2005, Lunar DPXtype, software Encore 2005, Rommelsdorf, Germany) body com-position analysis, and completed a familiarization session on aleg extension isoinertial machine (Righetto, Sao Paulo, Brazil) that involved performance of three sub-maximum sets of 8-10 repetitions. Three days later, subjects performed a $10 \mathrm{RM}$ test and following $72 \mathrm{~h}$ of rest they completed the $10 \mathrm{RM}$ test again to deter-mine test-retest reliability $(\mathrm{R}=$ 0.99). The test was terminated when voluntary concentric failure occurred (inability to perform a full range repetition of the movement as a consequence of fatigue).As described previously by Tibana et al.,19testing errors were minimized by the following strategies: (a) standardized instructions were given concerning all data collection procedures; (b)exercise technique and leg extension machine adjustments were standardized for each subject; and (c) subjects were given verbal encouragement throughout testing. Rest intervals of 3-5 min were instituted between 10 RM trials. Moreover, subjects were asked not to ingest any stimulants (e.g. caffeine) or perform any physical activity during the week prior to testing. 
Seven days following the $10 \mathrm{RM}$ tests, subjects completed an eccentric resistance exercise (ERE) protocol adapted from Willoughby et al.20Upon arrival at the lab, subjects began with a warm-up on a cycle ergometer for $10 \mathrm{~min}$ at $60 \mathrm{rpm}$ and $50 \mathrm{~W}$, followed by 10 leg extension repetitions at $50 \%$ of the $10 \mathrm{RM}$, and then a rest interval of 3-5 min. The ERE session was per-formed on the bilateral knee extension iso inertial machine with a load corresponding to $110 \%$ of the $10 \mathrm{RM}$. Subjects performed only the eccentric phase of the lift (2-3 s); at the end of each eccentric repetition, the researcher moved the load through the concentric portion of the range of motion to begin the next eccentric repetition. Subjects completed seven sets of 10 repetitions with a passive rest of 3 min between sets. The $10 \mathrm{RM}$ trials and ERE session were scheduled between 2:00 and 4:00 pm and were performed under standardized controlled room temperature. The knee extension exercise was chosen because the investigation of lower limb strength in the elderly is particularly important, considering that it is particularly affected by sarcopenia and loss offunctionality. 21

Blood samples were drawn from an antecubital vein by venipuncture to determine whole blood CK and IL-6 concentration pre- and 3, 24, and $48 \mathrm{~h}$ post exercise. CK concentration was determined by use of a commercially available Reflotron CKassay using the Reflotron system (Boehringer Mannheim GmbH, Mannheim, Germany). IL6 concentration was measured by Quantikine or Quantikine high sensitivity commercial enzyme-linked immunosorbent assay Kit (R\&D Systems, Minneapolis, MN, USA).The intra-assay coefficient of variation of the kits was 1.5-5.6\%for IL-6. The interassay coefficient of variation was $4.3-6.4 \%$ for IL-6. The measures for CK and IL-6 were performed in triplicate and averaged.

The data are expressed as means (95\% confidence interval).Shapiro-Wilk tests were applied to check for normality in distribution of the variables assessed. In case of non-normal distribution, the variables were log transformed to base e prior to analysis to approximate a normal distribution. The difference between base-line CK and peak CK concentration (the highest value achieved at $0,3,24$, or $48 \mathrm{~h}$ for each subject), or $\triangle \mathrm{CK}$, was considered the response following exposure to the ERE. The difference between baseline IL-6and peak IL-6 concentration (at $0,3,24$, or $48 \mathrm{~h}$ ), or $\triangle \mathrm{IL}-6$, was considered the response following exposure to the ERE. The achieved power of the sample size was determined using G*Power version3.1.5 (Kiel, Germany), based on the differences between baseline and peak concentrations of CK and IL- 6 between the HR and NR groups. For CK sample size ( $\mathrm{n}=90)$, the effect size $d$ was large and the power was 0.99 . For IL-6 sample size $(n=66)$, the effect size $d$ was also large and the power was 0.98 . The anthropometric data, CK and IL-6 peak values following the ERE were compared between the HR and NR groups using independent t-tests.

A mixed model ANOVA was used to compare the differences in CK and IL-6 concentration between groups at pre-exercise and over the course of $48 \mathrm{~h}$ postexercise. Compound sphericity was verified by the Mauchley test. When the assumption of sphericity was not met, the significance of F-ratios was adjusted 
according to the Greenhouse-Geisser procedure. Simple main effects were used to determine the difference between groups at each time point and to determine the difference between time points within each group. The level of significance was $\mathrm{p} \leq$ 0.05 and SPSS version 20.0 (Somers,NY, USA) software was used.

\begin{tabular}{|c|c|c|c|c|}
\hline & \multicolumn{2}{|l|}{ Creatine kinase (CK) } & \multicolumn{2}{|l|}{ Interleukin-6 (IL-6) } \\
\hline & $\mathrm{NR}(n=81)$ & $\operatorname{HR}(n=9)$ & $\mathrm{NR}(n=59)$ & $\operatorname{HR}(n=7)$ \\
\hline Age (year) & $69.8(68.4-71.1)$ & $66.2(62.3-70.1)$ & $68.6(67.0-70.2)$ & $75.4(71.0-79.8)^{*}$ \\
\hline Height (cm) & $152.6(151.3-153.9)$ & $153.8(147.6-160.1)$ & $152.8(151.1-154.4)$ & $151.0(144.8-157.2)$ \\
\hline Weight (kg) & $64.4(61.5-67.3)$ & $63.9(59.6-68.2)$ & $66.1(62.7-69.4)$ & $58.7(50.7-66.7)$ \\
\hline Body fat (\%) & $41.3(40.1-42.7)$ & $40.9(37.9-43.9)$ & $42.1(40.9-43.7)$ & $36.9(32.1-41.7)^{*}$ \\
\hline 10 RM (kg) & $36.5(34.5-38.4)$ & $40.0(31.3-48.7)$ & $37.6(35.3-40.0)$ & $34.4(23.4-45.4)$ \\
\hline$\Delta \mathrm{CK}(\mathrm{U} / \mathrm{l})$ & $22.5(17.3-27.8)$ & $233.4(150.0-316.9)^{\circ}$ & $38.3(22.2-54.4)$ & $37.7(-21.9-97.4)$ \\
\hline$\Delta \mathrm{lL}-6(\mathrm{pg} / \mathrm{ml})$ & $2.5(1.6-3.4)$ & $8.2(-0.7-3.12)$ & $1.6(1.2-2.1)$ & $15.6(4.6-26.6)^{*}$ \\
\hline
\end{tabular}

NR, normal responders; HR, high responders; $\Delta$, delta (change); $10 \mathrm{RM}$, ten repetitions maximum.

- Significantly different versus the NR group $(p \leq 0.05)$.

\section{Results}

Among the 90 subjects, nine $(10.0 \%)$ were classified as HR according to our predetermined statistical criteria for increases in serum CK concentration. Mean values for the NR and HR groups, based on CK responsiveness, are shown in Table 1 . The mean increase in serum CK concentration $(\Delta \mathrm{CK})$ for the HR group, $233.4(95 \% \mathrm{CI}$ : 150.0-316.9) U/l, was significantly greater $(\mathrm{p}<0.001)$ than the NR group, 22.5 (95\% CI: 17.3-27.8) U/l. All other variables (age, height, weight, percentage body fat, leg extension $10 \mathrm{RM}$, and $\Delta \mathrm{IL}-6$ ) were not significantly different between groups based on CK responsiveness. The time-course of serum CK in the HR and NR groups are shown in Fig. 1. There was a statistically significant interaction between the groups and time on serum CK concentration, $F(3.445,303.151)=18.275, p<0.001$. The CK concentration increased over the time following ERE for the HR group while no significant differences were noted for the NR group across time points. No significant differences between groups were noted in the base-line serum CK concentration (NR, 97.6 (95\% CI: 87.9-107.3) U/l and Fig. 1. Mean (95\% confidence interval) serum creatine kinase in normal (NR) and high (HR) responder groups prior to and at $0,3,24$ and $48 \mathrm{~h}$ following the eccentric resistance exercise session. *Significantly different versus the NR group ( $\mathrm{p} \leq$ 0.05).HR, 127.4 (95\% CI: 72.0182.9) $\mathrm{U} / \mathrm{l} ; \mathrm{p}=0.15)$. However, significantly greater serum $\mathrm{CK}$ concentration was noted for the HR group at 0 h,3 h, $24 \mathrm{~h}$ and $48 \mathrm{~h}$ following the ERE ( $<<0.001)$. The peak CK con-centration was significantly greater in the HR group versus the NRgroup (HR, 360.9 (95\% CI: 253.0-468.7) U/l and NR, 120.1 (95\% CI:110.4129.9) U/l; p $<0.001)$.

The IL- 6 concentration at baseline and following ERE was assessed in 66 women. Seven (10.6\%) were classified as HR accord-ing to our predetermined statistical criteria. Interestingly, only one subject met the criteria to be classified as HR for both CK and IL-6responsiveness. Mean values for the HR and NR groups, based onIL-6 responsiveness, are shown in Table 1 . The mean increase in the serum IL-6 (IL-6 $\Delta$ ) for the HR group, 15.6 (95\% CI: 4.6-26.6) pg/mlor approximately 850\%, 
was significantly greater ( $\mathrm{p}<0.001)$ than the NR group, $1.6(95 \% \mathrm{CI}: 1.2-2.1) \mathrm{pg} / \mathrm{ml}$ or approximately 55\%.When based on IL-6 responsiveness, age was significantly greater in the HR versus the NR group $(p=0.01)$ and the percentage bodyf at for the HR group was significantly less versus the NR group $(p=0.02)$. The time-course of serum IL-6 increases in the HR and NR groups are shown in Fig. 2. There was a statistically significant inter-action between the groups and time on serum IL-6 concentration, $F(1.976,80.998)=9.327, p<0.001$. The IL-6 concentration increased immediately following ERE and started to decrease over time until reaching baseline values $48 \mathrm{~h}$ after the ERE for the HR group, while no significant differences were noted for the NR group across time points. No significant differences between groups were noted in the baseline serum IL-6 concentration (NR, 5.3 (95\% CI: 3.57.0) pg/ml and HR, 3.8 (95\% CI: -6.2-13.7) pg/ml; p = 0.47). However, significantly greater serum IL-6 concentration was noted in the HR group at $0 \mathrm{~h}(\mathrm{p}=0.01)$ and 24 $h(p=0.02)$ following the ERE. There were no significant differences between groups in serum IL- 6 concentrationat $3 \mathrm{~h}(\mathrm{p}=0.08)$ and $48 \mathrm{~h}(\mathrm{p}=0.95)$ following the ERE. The peak IL-6concentration was significantly greater in the HR group versus the NR group (HR, 17.5 (95\% CI: 3.4-31.7) pg/ml and NR, 6.6 (95\% CI:5.08.2) $\mathrm{pg} / \mathrm{ml}$; $\mathrm{p}<0.001)$.

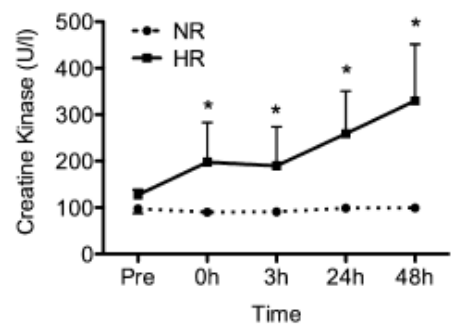

Fig. 1. Mean (95\% confidence interval) serum creatine kinase in normal (NR) and high (HR) responder groups prior to and at $0,3,24$ and $48 \mathrm{~h}$ following the eccentric resistance exercise session. "Significantly different versus the NR group $(p \leq 0.05)$.

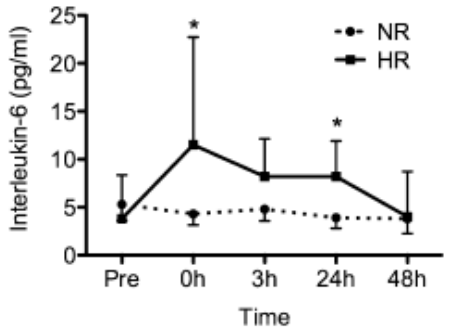

Fig. 2. Mean (95\% confidence interval) serum interleukin-6 in normal (NR) and high (HR) responder groups prior to and at 0, 3, 24 and $48 \mathrm{~h}$ following the eccentric resistance exercise session. *Significantly different versus the NR group $(p \leq 0.05)$.

\section{Discussion}

The key finding from the present study was the existence of an HR group for IL-6 in elderly obese women following the performance of ERE. The classification for the HR group in the present study was a $\Delta$ IL- 6 concentration $\geq 90$ th percentile or 7.5 $\mathrm{pg} / \mathrm{ml}$. Specifically, the percentage increase in IL-6 response for subjects below the 90th percentile was 55\%; whereas, the percentage increase for subjects above the 90th percentile (seven of the 66women) was 850\%. The HR group exhibited significantly greaterIL- 6 concentrations at $0 \mathrm{~h}$ and $24 \mathrm{~h}$ following the ERE versus the NR group, also the peak IL- 6 and $\Delta$ IL- 6 concentrations were significantly greater for the HR group versus the NR group. Moreover, when considering the $\Delta \mathrm{CK}$, nine of the 90 elderly women were included in the $\geq 90$ th percentile with CK concentrations at least96.3 U/l. The HR group exhibited significantly greater CK concentrations versus the NR group at $0 \mathrm{~h}, 3 \mathrm{~h}, 24 \mathrm{~h}$ and $48 \mathrm{~h}$ following the ERE. Additionally, the $\Delta \mathrm{CK}$ and peak CK concentrations were significantly greater for the HR group versus the NR group. These results confirmed our initial hypothesis. 
The increase in IL-6 following exercise may depend on factors such as age, body composition, and genetically expressed variation in the inflammatory response, including greater expression of toll-like receptor 4 (TLR4), that may induce greater transcription of inflammatory cytokines. 22 Thus, it is important to determine if any immune factors affect the transcription of other inflammatory immune factors and if a person that is considered a HR for one factor would be a HR for others. We found that the HR group for IL- 6 was older and had a lower body fat percentage as compared with the NR group. Although adipose tissue is an important endocrine organ responsible for the release of pro-inflammatorymarkers,23in the present study all women were elderly and obese. These two factors may account for an increased release of IL-6, as obesity and aging are associated with increased basal levels of IL6 and risk for chronic diseases.24It has been shown that IL-6 values $\geq 2.08 \mathrm{pg} / \mathrm{ml}$ was associated with a higher mortality rate of 50by 1000 per year in elderly individuals.25In the present study base-line values of IL-6 for both groups were high (3.8-4.3 pg/ml). Thus, attention should be given to HR elderly women when prescribing ERE, as they may reach significantly higher IL-6 levels $24 \mathrm{~h}$ following an exercise session.

The source of acute elevation in IL-6 immediately following ERE could be related to muscle contractions from muscle fibers, being considered a myokine. When released into the circulation,IL- 6 can promote metabolic modulations in several organs in a hormone-like fashion, such as hepatic glucose production during exercise or lipolysis in adipose tissue. Within skeletal muscle, IL-6 also activates metabolic pathways to increase glucose uptake and fat oxidation.26The acute release of IL-6 following exercise may also produce anti-inflammatory effects revealed by the production of the classical anti-inflammatory cytokines IL-1ra andIL-10.26

Interestingly, Bruunsgaard et al.27found that centenarians and81-year-old subjects have significantly higher plasma concentrations of IL-6 compared with a young control group (18-30 years).However, there was no difference in IL-6 levels between individuals of 55-65 years and octogenarians. Thus, it is possible that the difference in age between NR (68.6 years) versus HR (75.4 years) did not affect IL-6 results. Toft et al.18submitted ten elderly, all healthy and not taking any medication, to eccentric exercise consisting of 60 min of opposing the rotation of cycle ergometer pedals down to60 rpm. Post-exercise values of IL- 6 were found to be very similar to those observed in the NR group from the present study, while the HR group had higher values of IL- 6 in response to ERE.

Regarding CK, similar results were found in two previous studies that used resistance exercise, consisting of multiple exercises exercises for 3 sets at a $10 \mathrm{RM}$ load4or a single exercise for 4 sets at $85 \%$ of a 1repetition maximum (1RM).5Machado et al.5reported a very high CK response (i.e. approximately 2500 $\mathrm{U} / \mathrm{l}$ in an HR group) with a relatively low volume of work. In the present study, despite the ERE protocol, which is suggested to induce greater muscle damage,6peak values of CK increased to $361 \mathrm{U} / \mathrm{l}$ for the HR group. This difference 
may be due to distinct genders, age, genetics or physical training status; aspects that need to be elucidated in future studies. To note, the adopted protocol for ERE induced no alarming CK responses, which could be associated with excessive muscle dam-age and acute rhabdomyolysis.

Comparisons with other studies are very difficult due to a large degree of inconsistency in the exercise protocols utilized and the individuality in the CK response. Some studies were designed to induce muscle damage and were performed with unusual training methodologies, which may not reflect resistance training under practical conditions. The specific muscle group examined can also affect the magnitude of the CK response, as arm eccentric exercise induced larger decreases and a slower recovery of strength, and larger increases in blood markers of muscle damage (including $\mathrm{CK}$ ) versus leg exercise.28

Considering that only one subject met the criteria to be classified as HR for both CK and IL- 6 responsiveness, it can be hypothesized that these blood markers are unrelated, and that the mechanisms behind the rise in each are different. Also, IL-6 was measured in the circulation and it is possible that a different physiological environment could be present in the skeletal muscle, as Buford et al.29showed that skeletal muscle has a significant accumulation of transcripts for pro-inflammatory genes (TNF- $\alpha$, IL-1, IL-6and IL-8) $3 \mathrm{~h}$ following the completion of a resistance exercise bout. Moreover, if the increase in CK was a result of changes in membrane permeability rather than muscle damage, this might explain the lack of association between CK and IL-6. Alternatively, any association might have been confounded by the delay in the elimination of CK from the extracellular compartment due to the ratio of the enzyme's life-span to biological half-life.30

In the present study we utilized an ERE protocol because the reserve of eccentric strength, albeit variable, could be used, in combination with a greater magnitude of force development during eccentric muscle actions, allowing for a higher training intensity thus maximizing gains in muscle function in older adults.10 This reinforces the importance of ERE for frail elderly individuals exhibiting low muscular and cardiorespiratory fitness.

The present study had some limitations that should be considered, such as the genetic variability of the population examined, lack of morphological analysis of muscle damage and diet was not controlled (although they were advised to maintain their normal dietary intake). Moreover, the inclusion of a non-obese group of elderly women could be of value.

\section{Conclusions}

Only one subject was HR for both CK and IL-6, this implies that, although both blood markers are associated with muscular microtrauma, an individual presenting increased responsiveness of CK may not necessarily have a greater inflammatory response with respect to IL-6. Thus, we propose that multiple blood markers should 
be analyzed in response to exercise to improve the under-standing of the muscle damage response. Considering the longer time necessary to recover from an exercise session in elderly, these results could have implications for ERE prescription in that some individuals might require more days to recover and/or a lighter training, especially in the beginning stages of a resistance training program.

\section{Practical implications}

Both serum IL-6 and CK were higher for the HR versus NR, this information should be considered during the pre-participation screening process in exercise programs and training prescription to avoid excessive damage to muscle tissue and inflammation. Our data emphasize the need for specific safety cut-off point for circulating values of IL- 6 and CK response to ERE.A prudent approach for ERE prescription might be programming additional recovery days and/or lower intensity training, especially in the beginning stages of a program for HR individuals.

\section{References}

1. Barry BK, Carson RG. The consequences of resistance training for movement control in older adults. J Gerontol A Biol Sci Med Sci 2004; 59(7):730-754.

2. Paul AC, Rosenthal N. Different modes of hypertrophy in skeletal muscle fibers. J Cell Biol 2002; 156(4):751-760.

3. Dangott B, Schultz E, Mozdziak PE. Dietary creatine monohydrate supplementation increases satellite cell mitotic activity during compensatory hypertrophy. Int J Sports Med 2000; 21(1):13-16.

4. Machado M, Willardson JM. Short recovery augments magnitude of muscle damage in high responders. Med Sci Sports Exerc 2010; 42(7):1370-1374.

5. Machado M, Pereira R, Willardson JM. Short intervals between sets and individuality of muscle damage response. J Strength Cond Res 2012; 26(11):29462952.

6. Chen TC. Effects of a second bout of maximal eccentric exercise on muscle damage and electromyographic activity. Eur J Appl Physiol 2003; 89(2):115-121.

7. Sharp NC, Koutedakis Y. Sports and the overtraining syndrome: Immunological aspects. Brit Med Bull 1992; 48(3):518-533.

8. Vierck J, O’Reilly B, Hossner K et al. Satellite cell regulation following myotrauma caused by resistance exercise. Cell Biol Int 2000; 24(5):263-272.

9. Tomiya A, Aizawa T, Nagatomi R, et al. Myofibers express IL-6 after eccentric exercise. Am J Sports Med 2004; 32(2):503-508. 
10. Roig M, Macintyre DL, Eng JJ et al. Preservation of eccentric strength in older adults: evidence, mechanisms and implications for training and rehabilitation. Exp Gerontol 2010; 45(6):400-409.

11. Hortobagyi T. The positives of negatives: clinical implications of eccentric resistance exercise in old adults. J Gerontol A Biol Sci Med Sci 2003; 58(5):M417M418.

12. Reeves ND, Maganaris CN, Longo S et al. Differential adaptations to eccentric versus conventional resistance training in older humans. Exp Physiol 2009;94(7):825-833.

13. LaStayo PC, Pierotti DJ, Pifer J et al. Eccentric ergometry: increases in locomotor muscle size and strength at low training intensities. Am J Physiol Regul IntegrComp Physiol 2000; 278(5):R1282-R1288.

14. LaStayo PC, Ewy GA, Pierotti DD et al. The positive effects of negative work: increased muscle strength and decreased fall risk in a frail elderly population. JGerontol A Biol Sci Med Sci 2003; 58(5):M419-M424.

15. National. Institute of diabetes and digestive and kidney diseases. Understanding adult obesity (NIH Publ. no. 94-3680), Rockville, MD, National Institute of Health,1993.

16. Clarkson PM, Ebbeling C. Investigation of serum creatine kinase variability after muscle-damaging exercise. Clin Sci 1988; 75(3):257-261.

17. Yamashita K, Yoshioka T. Profiles of creatine kinase isoenzyme compositions insingle muscle fibres of different types. J Muscle Res Cell Motil 1991; 12(1):37-44.

18. Toft AD, Jensen LB, Bruunsgaard $\mathrm{H}$ et al. Cytokine response to eccentric exercise in young and elderly humans. Am J Physiol 2002; 283(1):C289-C295.

19. Tibana RA, Prestes J, Nascimento DC et al. Higher muscle performance in adolescents compared with adults after a resistance training session with different rest intervals. J Strength Cond Res 2012; 26(4):1027-1032.

20. Willoughby DS, McFarlin B, Bois C. Interleukin- 6 expression after repeated bouts of eccentric exercise. Int J Sports Med 2003; 24(1):15-21.

21. Baker BA, Cutlip RG. Skeletal muscle injury versus adaptation with aging: novel insights on perplexing paradigms. Exerc Sport Sci Rev 2010; 38(1):10-16. 
22. McFarlin BK, Flynn MG, Campbell WW et al. TLR4 is lower in resistance-trained older women and related to inflammatory cytokines. Med Sci Sports Exerc 2004;36(11):1876-1883.

23. Rasouli N, Kern PA. Adipocytokines and the metabolic complications of obesity. J Clin Endocrinol Metab 2008; 93(11 Suppl. 1):S64-S73.

24. Silva AO, Oliveira HB, Tavares AB et al. Comparison of inflammatory, metabolic, and anthropometric parameters in elderly women with and without insulin resistance. Res Aging 2012; 34(3):261-274.

25. Harris TB, Ferrucci L, Tracy RP et al. Associations of elevated interleukin-6and Creactive protein levels with mortality in the elderly. Am J Med 1999;106(5):506512.

26. Brandt C, Pedersen BK. The role of exercise-induced myokines in muscle homeostasis and the defense against chronic diseases. J Biomed Biotechnol 2010;5202:58.

27. Bruunsgaard $\mathrm{H}$, Andersen-Ranberg K, Jeune B et al. A high plasma concentration of TNF-alpha is associated with dementia in centenarians J Gerontol A Biol Sci MedSci 1999; 54(7):M357-M364.

28. Jamurtas AZ, Theocharis $\mathrm{V}$, Tofas $\mathrm{T}$ et al. Comparison between leg and arm eccentric exercises of the same relative intensity on indices of muscle damage. Eur JAppl Physiol 2005; 95(2-3):179-185.

29. Buford TW, Cooke MB, Willoughby DS. Resistance exercise-induced changes of inflammatory gene expression within human skeletal muscle. Eur J Appl Physiol2009; 107(4):463-471.

30. Scott JP, Sale C, Greeves JP. Cytokine response to acute running in recreationallyactive and endurance-trained men. Eur J Appl Physiol 2013; 113(7):1871-1882. 